\title{
Faunistic analysis of Ichneumonidae (Hymenoptera) in Guarana (Paullinia cupana) crop, with new records of genera for the Brazilian Amazon
}

\author{
Análise faunística de Ichneumonidae (Hymenoptera) na cultura de guaraná \\ (Paullinia cupana), com novos registros de gênero para a Amazônia brasileira
}

\author{
Nicanor Tiago Bueno Antunes' (1) (orcid.org/0000-0002-9101-340X) \\ Daniell Rodrigo Rodrigues Fernandes ${ }^{1 *}$ (1) (orcid.org/0000-0002-2208-6349)
}

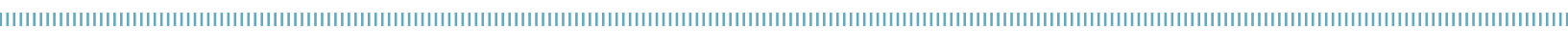

\begin{abstract}
Ichneumonidae are solitary parasitoids that mainly attack larvae and pupae of Lepidoptera, Coleoptera and Hymenoptera, which constitute important agricultural pests. The objective of the present paper was to characterize the assemblage of Ichneumonidae associated to the conventional and organic cultivation of Guarana (Paullinia cupana) crop in Manaus City (Amazonas State, Brazil) with faunistic analysis. The samples were collected at Empresa Brasileira de Pesquisa Agropecuária (EMBRAPA), using Malaise and Moericke traps, between September 2012 and February 2013. We collected 296 specimens of Ichneumonidae, represented by 14 subfamilies, 41 genera and 63 morphospecies. The faunistic analysis revealed the predominance of rare and less frequent genera $(54.2 \%$ in conventional and $47.1 \%$ in organic crop management). Venturia Schrottky was highlighted as superabundant, super frequent and constant ( $46.4 \%$ of the specimens) in conventional cultivation. In organic cultivation, Eiphosoma Cresson (15.6\% of the specimens), Venturia (15.1\%), Polycyrtidea Viereck (7\%), Stethantyx Townes (6\%), Enicospilus Stephens and Diapetimorpha Viereck (both 5.5\%), Polycyrtus Spinola and Podogaster Brullé (both 5\%) were more abundant, more frequent and constant. The observed differences reflect the type of crop management. Considering that organic crop management do not use agrochemicals, there is a greater diversification of the landscape. On the other hand, a reduction in richness and abundance has been observed in conventional crop management, due to its greater alteration and uniformity of the landscape. This study evidences a high number of rare, less frequent and accidental genera, and reveals the predominant groups in each crop cultivation system.
\end{abstract}

RESUMO: Ichneumonidae são parasitoides solitários e atacam principalmente larvas e pupas de Lepidoptera, Coleoptera e Hymenoptera, que constituem importantes pragas agrícolas. O objetivo do trabalho foi caracterizar a assembleia de Ichneumonidae associada ao cultivo de guaraná (Paullinia cupana) de manejo convencional e orgânico, em Manaus (Amazonas, Brasil) por meio de análise faunística. As coletas foram realizadas na EMBRAPA, com o uso de armadilhas Malaise e Moericke, entre setembro de 2012 e fevereiro de 2013. Foram coletados 296 exemplares de Ichneumonidae, em 14 subfamílias, 41 gêneros e 63 morfoespécies. A análise faunística revelou predominância de gêneros raros e pouco frequentes $(54,2 \%$ no manejo convencional e $47,1 \%$ no orgânico). Venturia Schrottky destacou-se no manejo convencional como superabundante, superfrequente e constante (46,4\% dos exemplares). No manejo orgânico, Eiphosoma Cresson (15,6\% dos exemplares), Venturia (15,1\%), Polycyrtidea Viereck (7\%), Stethantyx Townes (6\%), Enicospilus Stephens e Diapetimorpha Viereck (ambos 5,5\%), Polycyrtus Spinola e Podogaster Brullé (ambos 5\%) foram muito abundantes, muito frequentes e constantes. As diferenças observadas refletem o tipo de manejo da cultura. No manejo orgânico, por não utilizar agroquímicos, há uma maior diversificação da paisagem. Já no manejo convencional há uma redução da riqueza e abundância, por possuir maior perturbação e uniformidade da paisagem. Este estudo evidenciou o elevado número de gêneros raros, pouco frequentes e acidentais e expôs os grupos predominantes em cada sistema de manejo.

PALAVRAS-CHAVE: agroecossistemas; controle biológico; inimigos naturais; parasitoides.

KEYWORDS: agroecosystems; biological control; natural enemies; parasitoids. 


\section{INTRODUCTION}

Ichneumonidae (Hymenoptera: Ichneumonoidea) has about 25,000 valid species distributed along 42 subfamilies (YU et al., 2016; BENNETT et al., 2019). In general, they are solitary parasitoids and most of their species that have been biologically studied attack larvae and pupae of Lepidoptera, Coleoptera and Hymenoptera (GAULD, 1991). In Brazil, the family is represented by 1,019 species distributed in 233 genera (FERNANDES et al., 2020a).

Despite the ecological importance of Ichneumonidae, there is little knowledge about this group in most regions of Brazil, wherein the majority of studies were conducted in Atlantic Forest areas (KUMAGAI; GRAF, 2000; 2002; GUERRA; PENTEADO-DIAS, 2002; KUMAGAI, 2002; TANQUE et al., 2010; TANQUE et al., 2015), Cerrado (PÁDUA et al., 2014), Caatinga ( SHIMBORI et al., 2014; FERNANDES et al., 2019; FERNANDES et al., 2020b), and specific agroecosystems, such as rubber tree fauna (Hevea brasiliensis Muell. Arg.) (TEMPEST et al., 1998) and green dwarf coconut trees (Cocos nucifera L.) (COMÉRIO et al., 2012).

In general, the assemblage of crop-associated parasitoids is determined by the characteristics of the agroecosystem. Conventional cultivation systems simplify the physical structure of the agricultural landscape, with increased dependence on the use of inputs, which causes a reduction in the diversity of parasitoids in these agroecosystems (ESTRADA, 2008). However, in less disturbed agroecosystems, without the intensive use of agricultural inputs, parasitoid assembly is related to crop diversity, soil cover, weeds and adjacent crop vegetation (ESTRADA, 2008; SANDONATO et al., 2010).

In the Amazon forest, guarana (Paullinia cupana var. Sorbilis (Mart.) Ducke) is widely cultivated, encompassing an area of 8,029 hectares and production of 744 tons in 2017 , representing $22.6 \%$ of the country's guarana production (IBGE, 2017). However, the lack of basic studies (biological and ecological) of the fauna, especially regarding its natural enemies, such as predators and Hymenoptera parasitoids, delays the development of alternative techniques to conserve biodiversity and pest control associated to the cultivation of guarana.

Hence, the objective of the present paper was to characterize the Ichneumonidae assemblage associated to conventional and organic cultivation of guarana in Manaus City (Amazonas State, Brazil), and to determine the important genera associated to each crop management with faunistic analysis.

\section{MATERIAL AND METHODS}

A total of 12 collections were made between September 2012 and February 2013, during the flowering and fruiting phase of guarana. The collections were carried out at the premises of Brazilian Agricultural Research Corporation - Western Amazon - Amazonas State (Empresa Brasileira de Pesquisa Agropecuária - EMBRAPA), located in Northern Manaus (Amazonas State, Brazil) in two cultivation areas of guarana (Paullinia cupana var. Sorbilis (Mart.) Ducke): an organic crop management (2 $\left.2^{\circ} 3^{\prime} 29.14^{\prime \prime} \mathrm{S} / 59^{\circ} 58^{\prime} 45.80^{\prime \prime} \mathrm{W}\right)$ and a conventional crop management ( $\left.2^{\circ} 53^{\prime} 42.18^{\prime \prime} \mathrm{S} / 59^{\circ} 59^{\prime} 10.58^{\prime \prime W}\right)$.

The conventional crop management was established in 1986 and encompasses an area of 1.6 ha. During the study in this area, an insecticide was applied on January $1^{\text {st }}, 2013$. The organic crop management was established in 2003 with a total area of 3.9 ha. Both crop cultivations followed the management recommendations of PEREIRA (2005), with crops being cultivated at $5 \times 5 \mathrm{~m}$ spacing.

In each experimental area of cultivation, two collection points were selected, which were $60 \mathrm{~m}$ apart from each other. At each sampling point, one Malaise (TOWNES, 1972) and four Moericke (MOERICKE, 1951) traps were installed; with water and detergent solution arranged around the Malaise trap, at a distance of $5 \mathrm{~m}$. For more details on the sample design see SCHOENINGER et al. (2019).

Samples were collected every two weeks, where the traps were set for four days in the cultivation areas. The water and detergent solution of Moericke's traps was replaced every 24 hours to prevent the loss and deterioration of material.

The Ichneumonidae were identified at subfamily level, following PALACIO; WAHL (2006) and GAULD (2006). For generic identification, specific literature for each group was used. The distribution of genera throughout the Brazilian states was primarily defined according to the distribution presented in FERNANDES et al. (2020a) and complemented with the sporadic generic distributions present in literature. Subsequently, morphospecies identification was carried out based on their morphological characteristics. All individuals are deposited at the Invertebrate Collection of the National Institute for Amazonian Research, curated by Márcio Luiz de Oliveira.

Each crop management system was considered as a community with its own characteristics. The main groups in each crop management system were determined by the faunistic indices according to the method established by SILVEIRA-NETO et al. (1976). To determine the principal genera of each community, the indices of abundance, frequency and consistency were estimated for the genera of Ichneumonidae.

For the abundance index, we considered the total number of individuals sampled in the environment and calculated the confidence interval $(\mathrm{CI})$ of the means of genera at 5\% probability (SILVEIRA-NETO et al., 1976). Genera were classified into: more abundant, when the number of individuals was greater than the upper limit of the CI; abundant, when situated between the upper limits; common, when within the CI; dispersed, when the number of individuals was between the lower limit of the CI; and rare, when this 
number was lower than the lowest limit of the CI (LUDWIG; REYNOLDS, 1988).

Relative frequency was calculated by dividing the number of individuals of one genus by the total number of individuals collected, and the values obtained were expressed in percentage. The frequency index is estimated by distributing the values in histograms and compared based on the CI, with results being classified as less frequent, frequent and more frequent (DAJOZ, 1973).

For constancy, the percentage was calculated by multiplying the number of samples in which the genus was collected by 100 and then dividing it by the total number of samples (SILVEIRA-NETO et al., 1976). The genera were classified as: accidental, when collected from less than $25 \%$ of the samples; additional, when observed between 25 and $50 \%$ of the samples; and constant, when the genus was captured in more than $50 \%$ of the samples (SILVEIRA-NETO et al., 1976).

All faunistic indices were calculated by the Faunistic Analysis Software ANAFAU (MORAES et al., 2003), and the data obtained were used to determine the principal genera associated to crop management systems.

The richness was compared by generating an individual rarefaction curve, on the morphospecies abundance data in each crop management system. Standard error values (square root of variance) were converted to a $95 \%$ confidence interval and plotted in graphs. Abundance data from each management system were log-transformed to assess the proportion of abundance as a function of the number of genera sampled and plotted using MacArthur's broken stick model. Both graphs were generated by the paleontological statistics software package for education and data analysis - PAST, version 3.18 (HAMMER et al., 2001).

\section{RESULTS AND DISCUSSION}

A total of 296 specimens were collected, belonging to 14 subfamilies, and 41 genera. Out of these, 13 specimens (4.4\%) of Ichneumoninae were not identified at genera level and were counted as morphotypes. Out of 63 sampled morphospecies, 19 were captured in both crop management systems (Fig. 1A). 97 specimens were sampled in conventional management, which were classified into 10 subfamilies and 24 genera; and 199 specimens were collected in organic management, which were classified into 14 subfamilies and 34 genera (Table 1).

The rarefaction curve indicates that the species richness in organic crop management was higher than the richness observed in conventional crop management (Fig. 1B). However, it is noteworthy that both curves do not tend to stabilize, which may indicate a sampling deficiency for this insect group. Despite the efficiency of traps to capture Ichneumonidae (NOYES, 1989), the number of traps used per cultivation area might have been insufficient for representative sampling, which could have contributed to the observed high number of rare genera. Using more than one Malaise trap and four Moericke traps per cultivation area is suggested for a better representative sampling.

The results of faunistic analysis reveal that most of the sampled genera were considered rare, less frequent and accidental. In conventional crop management, among the 24 genera captured, $13(54.2 \%)$ genera were considered rare and less frequent, six $(25 \%)$ were common and frequent, four $(16.7 \%)$ were more abundant and more frequent and only Venturia Schrottky, 1902 (4.1\%) was super abundant and super frequent (Fig. 2A). In the organic crop management, out of 34 genera captured, 16 (47.1\%) were considered rare
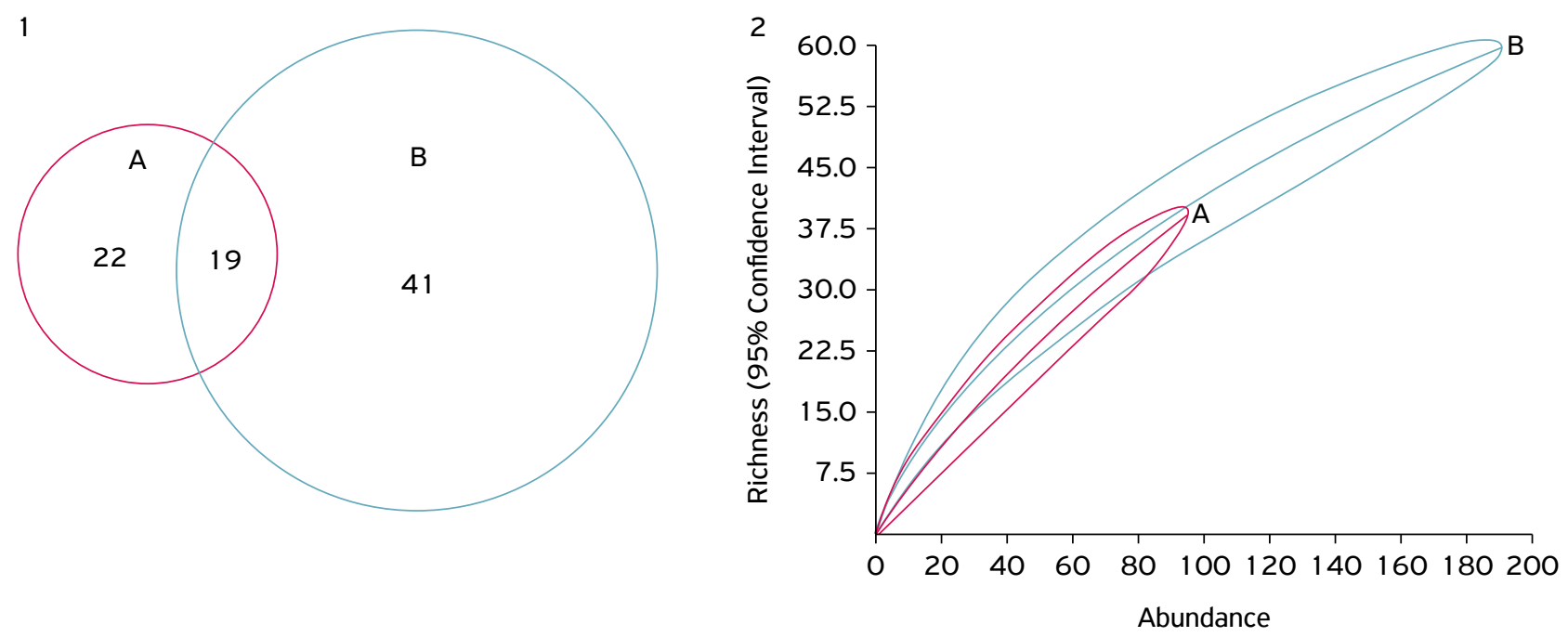

Figure 1. Ichneumonidae morphospecies sampled during the flowering period of Paullinia cupana in a conventional and organic crop management in EMBRAPA, Manaus (Amazonas, Brazil). (A) Conventional crop management system; (B) Organic crop management system. 1 - Venn diagram, diameter of the circles represents the richness between the managements. $2-$ Rarefaction curve. 
Table 1. Faunistic analysis applied to Ichneumonidae genera associated with conventional and organic cultivation of Paullinia cupana of EMBRAPA in Manaus (Amazonas, Brazil).

\begin{tabular}{|c|c|c|c|c|c|c|c|c|c|c|}
\hline \multirow{2}{*}{ Taxon } & \multicolumn{5}{|c|}{ Conventional } & \multicolumn{5}{|c|}{ Organic } \\
\hline & N.I & F (\%) & A & $\mathbf{F}$ & C & N.I & F (\%) & A & $\mathbf{F}$ & C \\
\hline \multicolumn{11}{|l|}{ Anomaloninae } \\
\hline Agrypon Forster, 1860 & - & - & - & - & - & 1 & 0,5 & $r$ & LF & Z \\
\hline Podogaster Brullé, 1846 & 7 & 7,2 & ma & MF & $\mathrm{Y}$ & 10 & 5 & ma & MF & W \\
\hline
\end{tabular}

Banchinae

\begin{tabular}{|c|c|c|c|c|c|c|c|c|c|c|}
\hline Lissonota Gravenhorst, 1829 & 1 & 1 & $r$ & LF & Z & - & - & - & & - \\
\hline Meniscomorpha Schmiedeknecht, 1907 & - & - & - & - & - & 1 & 0,5 & $r$ & LF & Z \\
\hline \multicolumn{11}{|l|}{ Campopleginae } \\
\hline Charops Holmgren, 1859 & - & - & - & - & - & 2 & 1 & $r$ & LF & Z \\
\hline Microcharops Roman, 1910 & - & - & - & - & - & 3 & 1,5 & d & LF & $\mathrm{Y}$ \\
\hline Venturia Schrottky, 1902 & 45 & 46,4 & sa & SF & W & 30 & 15,1 & ma & MF & W \\
\hline
\end{tabular}

Cremastinae

Creagrura Townes, 1971

Eiphosoma Cresson, 1865

Eutanygaster Cameron, 1911

Xiphosomella Szepligeti, 1905

$\begin{array}{cccccccccc}- & - & - & - & - & 1 & 0,5 & \mathrm{r} & \text { LF } & \mathrm{Z} \\ 2 & 2,1 & \mathrm{C} & \mathrm{F} & \mathrm{Z} & 31 & 15,6 & \mathrm{ma} & \mathrm{MF} & \text { W } \\ - & - & - & - & - & 4 & 2 & \mathrm{c} & \mathrm{F} & \mathrm{Y} \\ 3 & 3,1 & \mathrm{C} & \mathrm{F} & \mathrm{Z} & 6 & 3 & \mathrm{c} & \mathrm{F} & \mathrm{Y}\end{array}$

Cryptinae

\begin{tabular}{|c|c|c|c|c|c|c|c|c|c|c|}
\hline Acerastes Cushman, 1929 & 1 & 1 & $r$ & LF & Z & 2 & 1 & $r$ & LF & Z \\
\hline Agonocryptus Cushman, 1929 & 1 & 1 & $r$ & LF & Z & - & - & - & & - \\
\hline Cryptanura Brullé, 1846 & - & - & - & - & - & 1 & 0,5 & $r$ & LF & Z \\
\hline Debilos Townes, 1966 & 5 & 5,2 & ma & MF & Z & 6 & 3 & c & $\mathrm{F}$ & $\mathrm{Y}$ \\
\hline Diapetimorpha Viereck, 1913 & 1 & 1 & $r$ & LF & Z & 11 & 5,5 & ma & MF & W \\
\hline Glodianus Cameron, 1902 & - & - & - & - & - & 1 & 0,5 & $r$ & LF & Z \\
\hline Golbachiela Townes, 1970 & 1 & 1 & $r$ & LF & Z & - & - & - & & - \\
\hline Lamprocryptus Schmiedeknecht, 1904 & - & - & - & - & - & 1 & 0,5 & $r$ & LF & Z \\
\hline Lymeon Forster, 1869 & 2 & 2,1 & c & $\mathrm{F}$ & Z & 3 & 1,5 & $d$ & LF & $\mathrm{Y}$ \\
\hline Mallochia Viereck, 1912 & - & - & - & - & - & 2 & 1 & $r$ & LF & Z \\
\hline Messatoporus Cushmam, 1929 & 1 & 1 & $r$ & LF & Z & - & - & - & & - \\
\hline Photocryptus Viereck, 1913 & - & - & - & - & - & 6 & 3 & c & $\mathrm{F}$ & $\mathrm{Y}$ \\
\hline Polycyrtidea Viereck, 1913 & 1 & 1 & $r$ & LF & Z & 14 & 7 & ma & MF & w \\
\hline Polycyrtus Spinola, 1840 & 3 & 3,1 & c & $\mathrm{F}$ & Z & 10 & 5 & ma & MF & W \\
\hline Rhinium Townes, 1966 & 3 & 3,1 & c & $\mathrm{F}$ & Z & 2 & 1 & $r$ & LF & Z \\
\hline Toechorychus Townes, 1946 & 1 & 1 & $r$ & LF & $Z$ & - & - & - & & - \\
\hline Whymperia Cameron, 1913 & - & - & - & - & - & 1 & 0,5 & $r$ & LF & Z \\
\hline \multicolumn{11}{|l|}{ Ctenopelmatinae } \\
\hline Lathrolestes Forster, 1869 & - & - & - & - & - & 2 & 1 & $r$ & LF & Z \\
\hline \multicolumn{11}{|l|}{ Ichneumoninae } \\
\hline Ichneumoninae (unidentified genera) & 6 & 6,2 & $\mathrm{ma}$ & MF & $\mathrm{Y}$ & 7 & 3,5 & c & $\mathrm{F}$ & $\mathrm{Y}$ \\
\hline Joppa Fabricius, 1804 & 3 & 3,1 & c & $\mathrm{F}$ & Z & - & - & - & & - \\
\hline \multicolumn{11}{|l|}{ Labeninae } \\
\hline Apechoneura Kriechbaumer, 1890 & 1 & 1 & $r$ & LF & z & - & - & - & & - \\
\hline Grotea Cresson, 1864 & 1 & 1 & $r$ & LF & Z & 1 & 0,5 & $r$ & LF & Z \\
\hline
\end{tabular}


Table 1. Continuation.

\begin{tabular}{|c|c|c|c|c|c|c|c|c|c|c|}
\hline \multirow{2}{*}{ Taxon } & \multicolumn{5}{|c|}{ Conventional } & \multicolumn{5}{|c|}{ Organic } \\
\hline & N.I & F (\%) & A & $\mathbf{F}$ & C & N.I & $F(\%)$ & A & $\mathbf{F}$ & C \\
\hline \multicolumn{11}{|l|}{ Mesochorinae } \\
\hline Mesochorus Gravenhorst, 1829 & 1 & 1 & $r$ & LF & Z & 2 & 1 & $r$ & LF & Z \\
\hline \multicolumn{11}{|l|}{ Nesomesochorinae } \\
\hline Nonnus Cresson, 1874 & - & - & - & - & - & 3 & 1,5 & $d$ & LF & $\mathrm{Y}$ \\
\hline \multicolumn{11}{|l|}{ Ophioninae } \\
\hline Enicospilus Stephens, 1835 & 1 & 1 & $r$ & LF & Z & 11 & 5,5 & ma & MF & W \\
\hline \multicolumn{11}{|l|}{ Orthocentrinae } \\
\hline Eusterinx Forster, 1869 & - & - & - & - & - & 4 & 2 & c & $\mathrm{F}$ & $\mathrm{Y}$ \\
\hline Orthocentrus Gravenhorst, 1829 & 1 & 1 & $r$ & LF & Z & 5 & 2,5 & c & $\mathrm{F}$ & $\mathrm{Y}$ \\
\hline Plectiscidea Viereck, 1914 & - & - & - & - & - & 1 & 0,5 & $r$ & LF & Z \\
\hline \multicolumn{11}{|l|}{ Tersilochinae } \\
\hline Stethantyx Townes, 1971 & 5 & 5,2 & ma & MF & $\mathrm{Y}$ & 12 & 6 & ma & MF & W \\
\hline \multicolumn{11}{|l|}{ Tryphoninae } \\
\hline Netelia Gray, 1860 & - & - & - & - & - & 2 & 1 & $r$ & LF & Z \\
\hline
\end{tabular}

N.I: number of individuals sampled. $\mathrm{F}(\%)$ : frequency; A: abundance index: sa: superabundant; ma: more abundant; a: abundant; c: common; d: dispersed; r: rare; F: frequency index: SF: superfrequent; MF: more frequent; F: frequent; LF: less frequent; C: constancy: W: constant; $\mathrm{Y}$ : additional; Z: accidental.
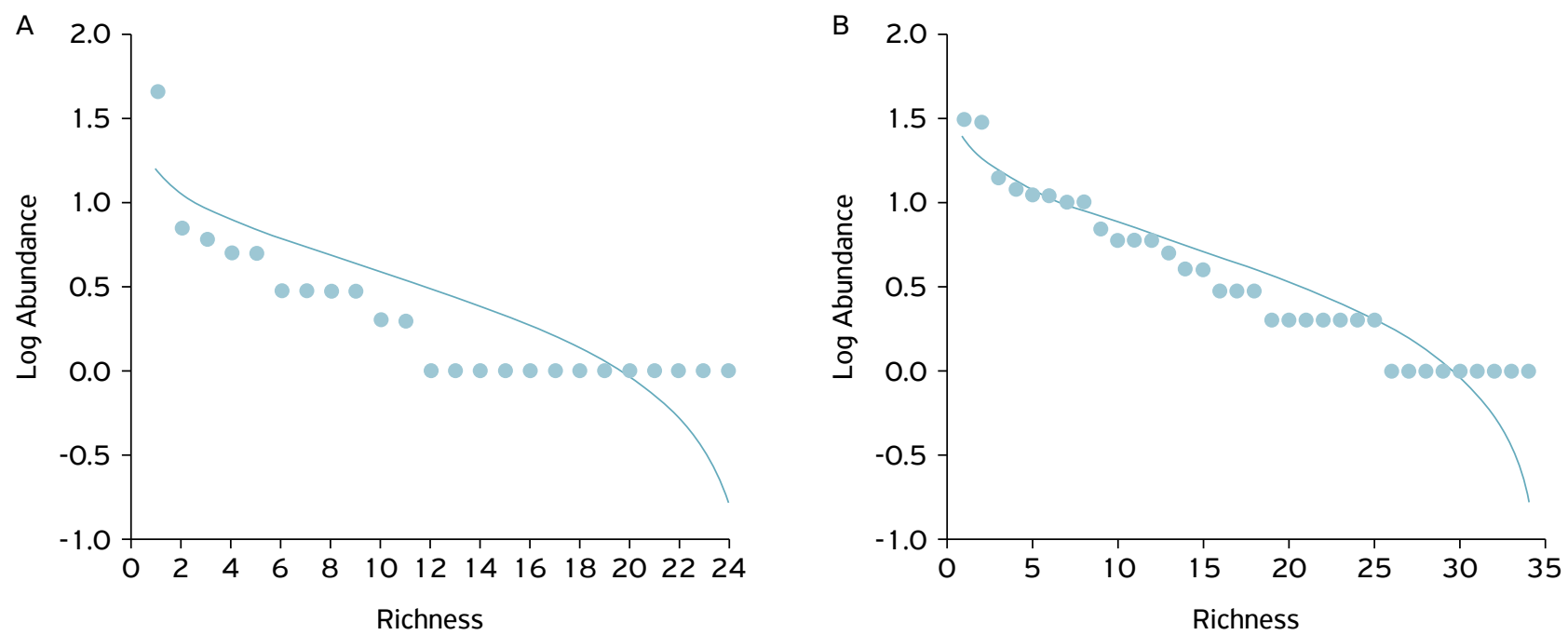

Figure 2. Abundance of Ichneumonidae genera, sampled during the flowering period of Paullinia cupana from EMBRAPA, Manaus (Amazonas, Brazil), in broken stick model. In (A), genera sampled in conventional crop management system; in (B), genera sampled in organic crop management system.

and three $(8.8 \%)$ were considered dispersed. When combined, these 19 genera were less frequent; of the remaining 15 genera, seven $(20.6 \%)$ were common and frequent, and eight were (23.5\%) more abundant and more frequent (Fig. 2B).

Ichneumonidae assemblage surveys are scarce in agroecosystems of Brazil (PERIOTO et al., 2002), and few studies have been carried out on rubber tree Hevea brasiliensis (Euphorbiaceae) (TEMPEST et al., 1998) and green dwarf coconut tree Cocos nucifera (Arecaceae) (COMÉRIO et al.,
2012) plantations. Thus, this serves as the pioneer study in the field of Ichneumonidae assemblage in guarana cultivation.

The greater richness and abundance found in organic management is related to the characteristics of this type of cultivation. Organic management does not use agrochemicals, which allows a greater landscape diversification. However, the general tendency of conventional management is to reduce the richness and abundance of both native flora and fauna, because of the associated alterations due to the use of agrochemicals, soil 
management and the uniformity of landscape (PFIFFNER; LUKA, 2000).

This major alteration in conventional crop management results in a higher incidence of rare, less frequent and accidental genera. In organic management, due to greater landscape diversification, there is a more homogeneous distribution in the analyzed indices. However, in both managements there was a predominance of rare genera. Studies conducted in tropical and subtropical forests have found that the percentage of rare species in different arthropod collections is about 60\% (NOYES, 1989; CODDINGTON et al., 2009; HOLDEFER; GARCIA, 2015), close to the value obtained in this study for Ichneumonidae. NOVOTNÝ; BASSET (2000), whereas studying an elevated rate of rare species in herbivorous insect communities, suggested that species richness is influenced by a constant influx of insects from adjacent plants. Since Ichneumonidae has a wide diversity of hosts, these parasitoids may have migrated to the cultivation area following this influx.

The major groups determined by the faunistic analysis were Venturia Schrottky, 1902, which was superabundant, super frequent and constant, with $46.4 \%$ of the total specimens collected in conventional management. In organic management, the genera Eiphosoma Cresson, 1865 (15.6\% of the specimens), Venturia (15.1\% of the specimens), Polycyrtidea Viereck, 1913 (7\% of the specimens), Stethantyx Townes 1971 (6\% of the specimens), Enicospilus Stephens, 1835 and Diapetimorpha
Viereck, 1913 (both 5.5\% of specimens), Polycyrtus Spinola, 1840 and Podogaster Brullé, 1846 (both 5\% of specimens) had more abundant, more frequent and constant indices.

The predominance and constancy of these groups in organic and conventional managements may be occurring due to the availability of hosts (Table 2). According to GONDIN (1984), Lepidoptera and Coleoptera represented $38 \%$ of guarana floral visitors, and these are the most frequent hosts of Ichneumonidae. Only Brachycyrtinae, Diplazontinae, Neorhacodinae and Orthocentrinae do not include these orders among their hosts (FERNÁNDEZ-TRIANA; RAVELO, 2007).

Based on the recorded distribution of genera in Brazil, it was verified that Plectiscidea Viereck, 1914 is registered for the first time in the country; and Acerastes Cushman, 1929, Agonocryptus Cushman, 1929, Creagrura Townes, 1971, Eusterinx Forster, 1869, Eutanygaster Cameron, 1911, Glodianus Cameron, 1902, Golbachiela Townes, 1970, Lathrolestes Forster, Lissonota Gravenhorst, 1869, Mallochia Viereck, 1912, Meniscomorpha Schmie-deknecht, 1907, Mesochorus Gravenhorst, 1829, Orthocentrus Gravenhorst, 1829, Polycyrtidea Viereck, 1913, Rhinium Townes, 1966, Stethantyx, and Venturia are recorded for the first time in Amazonas State (Table 3).

In Brazil, currently 233 genera are recorded in 28 subfamilies, and for the state of Amazonas, 52 genera in 14 subfamilies (FERNANDES et al., 2020a). This study increases the known genera in Amazonas from 52 to 70, and records

Table 2. Principal genera of Ichneumonidae, sampled during the flowering period of Paullinia cupana from EMBRAPA, in Manaus (Amazonas, Brazil) and their hosts.

\begin{tabular}{|c|c|c|}
\hline Taxon & Hosts & References \\
\hline \multicolumn{3}{|l|}{ Anomaloninae } \\
\hline Podogaster Brullé, 1846 & $\begin{array}{l}\text { Larvae of Saturnidae } \\
\text { and Pyralidae (Lepidoptera) }\end{array}$ & GONZÁLES-MORENO; BORDERA (2013) \\
\hline \multicolumn{3}{|l|}{ Campopleginae } \\
\hline Venturia Schrottky, 1902 & Larvae of Pyraloidea (Lepidoptera) & $\begin{array}{l}\text { GAULD (1984); SANDONATO et al. (2010); } \\
\text { GONZÁLEZ; BERRIO (2015) }\end{array}$ \\
\hline \multicolumn{3}{|l|}{ Cremastinae } \\
\hline Eiphosoma Cresson, 1865 & $\begin{array}{l}\text { Larvae of Pyralidae } \\
\text { and Noctuidae (Lepidoptera) }\end{array}$ & $\begin{array}{c}\text { GAULD (2000); } \\
\text { FERNÁNDEZ-TRIANA; RAVELO (2007) }\end{array}$ \\
\hline \multicolumn{3}{|l|}{ Cryptinae } \\
\hline $\begin{array}{l}\text { Diapetimorpha Viereck, } \\
1913\end{array}$ & Pupae of Noctuidae (Lepidoptera) & HANSON; GAULD (2006) \\
\hline Polycyrtidea Viereck, 1913 & No host records. & 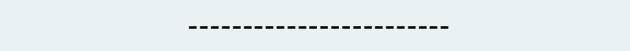 \\
\hline Polycyrtus Spinola, 1840 & Crambidae (Lepidoptera) & KASPARYAN; RUÍZ-CANCINO (2008) \\
\hline \multicolumn{3}{|l|}{ Ophioninae } \\
\hline Enicospilus Stephens, 1835 & Larvae of Lepidoptera & $\begin{array}{l}\text { GAULD; MITCHELL (1978); GAULD; } \\
\text { MITCHELL (1981); GAULD (1988); } \\
\text { FERNANDES et al. (2014) }\end{array}$ \\
\hline \multicolumn{3}{|l|}{ Tersilochinae } \\
\hline Stethantyx Townes, 1971 & Nitidulidae and Curculionidae (Coleoptera) & WILLIAMS et al. (1984); KHALAIM et al. (2013) \\
\hline
\end{tabular}


Ctenopelmatinae, Mesochorinae, Orthocentrinae and Tersilochinae, for the first time in the state of Amazonas, extending the sampled subfamilies from 14 to 18 .

The number of sampled genera demonstrates the potential of the Amazon region for studies of group diversity. Moreover, the new records evidenced the absence of studies in this region as well as in the country. A survey on the Ichneumonidae fauna conducted in Northeastern Brazil identified 17 genera, of which 14 were new records for that region, and highlighted the difficulty of specimen identification, due to lack of literature that includes Brazilian fauna. (SHIMBORI et al., 2014). In a survey conducted in Bahia

Table 3. Number of morphospecies and distribution of Ichneumonidae genera associated with conventional and organic cultivation of Paullinia cupana in EMBRAPA, Manaus (Amazonas, Brazil).

\begin{tabular}{|c|c|c|c|}
\hline Taxon & Morphospecies & Distribution & References \\
\hline \multicolumn{4}{|l|}{ Anomaloninae } \\
\hline Agrypon Forster, 1860 & 1 & AM, PA, RS & FERNANDES et al. (2020a) \\
\hline Podogaster Brulle, 1846 & 2 & $\begin{array}{l}\text { AM, ES, MS, PA, PR, SC, } \\
\text { SP }\end{array}$ & $\begin{array}{l}\text { AZEVEDO et al. (2015); FERNANDES et al. } \\
\text { (2020a); SHIMBORI et al. (2017) }\end{array}$ \\
\hline \multicolumn{4}{|l|}{ Banchinae } \\
\hline Lissonota Gravenhorst, 1829 & 1 & $\mathrm{AM}^{*}, \mathrm{BA}, \mathrm{PR}$ & $\begin{array}{l}\text { KUMAGAI; GRAF (2000); FERNANDES et al. } \\
\text { (2019) }\end{array}$ \\
\hline $\begin{array}{l}\text { Meniscomorpha } \\
\text { Schmiedeknecht, } 1907\end{array}$ & 1 & $\mathrm{AM}^{*}, \mathrm{PR}, \mathrm{SP}$ & FERNANDES et al. (2020a) \\
\hline \multicolumn{4}{|l|}{ Campopleginae } \\
\hline Charops Holmgren, 1859 & 1 & $\begin{array}{c}\text { AM, AC, BA, CE, ES, MA, } \\
\text { MG, MS, MT, PA, PB, PE, PI, } \\
\text { PR, RJ, RN, RR, RS, SC, SP }\end{array}$ & $\begin{array}{l}\text { AZEVEDO et al. (2015); FERNANDES et al. } \\
\text { (2019); FERNANDES et al. (2020a); KUMAGAl; } \\
\text { GRAF (2000); SANDONATO et al. (2010) }\end{array}$ \\
\hline Microcharops Roman, 1910 & 1 & $\begin{array}{l}\text { AM, BA, CE, DF, ES, GO, } \\
\text { MS, MT, PA, PE, PR, RJ, } \\
\text { RO, SC, SP }\end{array}$ & $\begin{array}{l}\text { FERNANDES et al. (2020a); SHIMBORI et al. } \\
\qquad(2017)\end{array}$ \\
\hline Venturia Schrottky, 1902 & 3 & $\mathrm{AM}^{*}, \mathrm{BA}, \mathrm{MS}, \mathrm{PR}, \mathrm{RS}, \mathrm{SP}$ & $\begin{array}{c}\text { FERNANDES et al. (2019); FERNANDES et al. } \\
\text { (2020a); KUMAGAI; GRAF (2000); SHIMBORI } \\
\text { et al. (2017) }\end{array}$ \\
\hline \multicolumn{4}{|l|}{ Cremastinae } \\
\hline Creagrura Townes, 1971 & 1 & $\mathrm{AM}^{*}, \mathrm{ES}, \mathrm{RJ}$ & $\begin{array}{l}\text { AZEVEDO et al. (2015); FERNANDES et al. } \\
\qquad(2020 a)\end{array}$ \\
\hline Eiphosoma Cresson, 1865 & 5 & $\begin{array}{l}\text { AM, BA, CE, ES, GO, MA, } \\
\text { MG, MS, MT, PA, PB, PE, } \\
\text { PI, PR, RJ, RN, SP }\end{array}$ & $\begin{array}{l}\text { FERNANDES et al. (2019); Fernandes et al. } \\
\text { (2O20a); KUMAGAI; GRAF (2000); SHIMBORI } \\
\text { et al. (2014); SHIMBORI et al. (2017) }\end{array}$ \\
\hline Eutanygaster Cameron, 1911 & 1 & $\mathrm{AM}^{*}, \mathrm{ES}, \mathrm{SP}$ & $\begin{array}{l}\text { AZEVEDO et al. (2015); FERNANDES et al. } \\
\text { (2020a) }\end{array}$ \\
\hline Xiphosomella Szepligeti, 1905 & 6 & $\begin{array}{l}\text { AM, BA, SC, ES, } \\
\text { PA, PR, RN }\end{array}$ & $\begin{array}{l}\text { Trocar por: AZEVEDO et al. (2015); } \\
\text { FERNANDES et al. (2019); FERNANDES } \\
\text { et al. (2020a); FERNANDES et al. (2020b); } \\
\text { KUMAGAI; GRAF (2000) }\end{array}$ \\
\hline \multicolumn{4}{|l|}{ Cryptinae } \\
\hline Acerastes Cushman, 1929 & 2 & $\mathrm{AM}^{*}, \mathrm{ES}, \mathrm{PR}, \mathrm{RJ}, \mathrm{SC}$ & $\begin{array}{l}\text { AZEVEDO et al. (2015); FERNANDES et al. } \\
\text { (2020a); KUMAGAl; GRAF (2000) }\end{array}$ \\
\hline Agonocryptus Cushman, 1929 & 1 & $\begin{array}{c}\mathrm{AM}^{*}, \mathrm{CE}, \mathrm{ES}, \mathrm{GO}, \mathrm{MG}, \mathrm{MT} \\
\mathrm{PA}, \mathrm{PB}, \mathrm{PE}, \mathrm{PR}, \mathrm{RJ}, \mathrm{RO} \\
\text { SC, SP }\end{array}$ & $\begin{array}{l}\text { FERNANDES et al. (2020a), KUMAGAI; Graf } \\
\qquad(2000)\end{array}$ \\
\hline \multirow[t]{2}{*}{ Cryptanura Brulle, 1846} & 1 & $\begin{array}{l}\text { AM, BA, ES, MG, PI, PR, } \\
\text { RJ, RN, SC, SP }\end{array}$ & $\begin{array}{l}\text { AZEVEDO et al. (2015); FERNANDES } \\
\text { et al. (2019); FERNANDES et al. (2020a); } \\
\text { KUMAGAI; GRAF (2000) }\end{array}$ \\
\hline & & & Continue. \\
\hline
\end{tabular}


Table 3. Continuation.

\begin{tabular}{|c|c|c|c|}
\hline Taxon & Morphospecies & Distribution & References \\
\hline Debilos Townes, 1966 & 2 & $\begin{array}{c}\text { AC, AM, BA, ES, MG, PA, } \\
\text { RJ, RO }\end{array}$ & $\begin{array}{l}\text { FERNANDES et al. (2020a); SCHERRER; } \\
\text { AGUIAR (2012) }\end{array}$ \\
\hline Diapetimorpha Viereck, 1913 & 4 & AM, PR, RJ, SC & $\begin{array}{l}\text { FERNANDES et al. (2020a); } \\
\text { KUMAGAI; GRAF (2000) }\end{array}$ \\
\hline Glodianus Cameron, 1902 & 1 & $\mathrm{AM}^{*}, \mathrm{PR}, \mathrm{RJ}$ & $\begin{array}{l}\text { FERNANDES et al. (2020a); } \\
\text { KUMAGAI; GRAF (2000) }\end{array}$ \\
\hline Golbachiela Townes, 1970 & 1 & $\mathrm{AM}^{*}, \mathrm{ES}$ & AZEVEDO et al. (2015) \\
\hline $\begin{array}{l}\text { Lamprocryptus } \\
\text { Schmiedeknecht, } 1904\end{array}$ & 1 & AM, ES, MT, PI, RS & $\begin{array}{l}\text { AZEVEDO et al. (2015); } \\
\text { FERNANDES et al. (2020a) }\end{array}$ \\
\hline Lymeon Forster, 1869 & 3 & AM, MG, PA, PI, PR, SC, RJ & $\begin{array}{l}\text { FERNANDES et al. (2020a); } \\
\text { KUMAGAl; GRAF (2000) }\end{array}$ \\
\hline Mallochia Viereck, 1912 & 1 & $\mathrm{AM}^{*}, \mathrm{PR}, \mathrm{SC}$ & $\begin{array}{l}\text { FERNANDES et al. (2020a); } \\
\text { KUMAGAl; GRAF (2000) }\end{array}$ \\
\hline Messatoporus Cushmam, 1929 & 1 & $\begin{array}{l}\text { AM, BA, ES, GO, MG, MT, } \\
\text { PA, PE, PR, RJ, RN, RO, } \\
\text { RS, SC, SP }\end{array}$ & $\begin{array}{l}\text { AZEVEDO et al. (2015); FERNANDES et al. } \\
\qquad(2020 \mathrm{a})\end{array}$ \\
\hline Photocryptus Viereck, 1913 & 1 & $\begin{array}{l}\text { AM, AP, ES, GO, MG, PA, } \\
\text { PR, RJ, RR, SP, SC }\end{array}$ & $\begin{array}{l}\text { AGUIAR; SANTOS (2009); FERNANDES et al. } \\
\text { (2020a); KUMAGAI; GRAF (2000) }\end{array}$ \\
\hline Polycyrtidea Viereck, 1913 & 2 & $\mathrm{AM}^{*}, \mathrm{ES}, \mathrm{RN}$ & AZEVEDO et al. (2015); FERNANDES et al. (2020b) \\
\hline Polycyrtus Spinola, 1840 & 4 & $\begin{array}{l}\text { AM, MG, PI, PA, PR, RJ, } \\
\text { RS, SC, SP }\end{array}$ & FERNANDES et al. (2020a) \\
\hline Rhinium Townes, 1966 & 5 & $\mathrm{AM}^{*}, \mathrm{ES}$ & AZEVEDO et al. (2015) \\
\hline Toechorychus Townes, 1946 & 1 & $\begin{array}{l}\text { AM, AP, BH, ES, GO, MG, } \\
\text { MT, PA, PR, RJ, RO, SC, SP }\end{array}$ & $\begin{array}{l}\text { FERNANDES et al. (2020a); } \\
\text { TEDESCO; AGUIAR (2013) }\end{array}$ \\
\hline Whymperia Cameron, 1913 & 1 & AM, PA & FERNANDES et al. (2020a) \\
\hline \multicolumn{4}{|l|}{ Ctenopelmatinae } \\
\hline Lathrolestes Forster, 1869 & 1 & $\mathrm{AM}^{*}, \mathrm{MG}$ & FERNANDES et al. (2020a) \\
\hline \multicolumn{4}{|l|}{ Ichneumoninae } \\
\hline Ichneumoninae & 7 & $\begin{array}{l}\text { AM, BA, ES, GO, MG, PA, } \\
\text { PE, PI, PR, RJ, RS, SC, SP }\end{array}$ & FERNANDES et al. (2020a) \\
\hline Joppa Fabricius, 1804 & 1 & $\begin{array}{l}\text { AM, BA, ES, GO, MG, PA, } \\
\text { PE, PI, PR, RJ, RS, SC, SP }\end{array}$ & $\begin{array}{l}\text { FERNANDES et al. (2020a); } \\
\text { KUMAGAI; Graf (2000) }\end{array}$ \\
\hline \multicolumn{4}{|l|}{ Labeninae } \\
\hline $\begin{array}{l}\text { Apechoneura } \\
\text { Kriechbaumer, } 1890\end{array}$ & 1 & AM, ES, MG & FERNANDES et al. (2020) \\
\hline Grotea Cresson, 1864 & 1 & $\begin{array}{l}\text { AM, BA, ES, GO, MG, PI, } \\
\text { PR, RJ, SC, SP }\end{array}$ & $\begin{array}{l}\text { AZEVEDO et al. (2015); FERNANDES et al. } \\
\text { (2019); Fernandes et al. (2020a) }\end{array}$ \\
\hline \multicolumn{4}{|l|}{ Mesochorinae } \\
\hline Mesochorus Gravenhorst, 1829 & 3 & $\begin{array}{l}\mathrm{AM}^{*}, \mathrm{BA}, \mathrm{GO}, \mathrm{MG}, \mathrm{PA}, \mathrm{PB} \\
\mathrm{PE}, \mathrm{PI}, \mathrm{PR}, \mathrm{RJ}, \mathrm{RN}, \mathrm{RR} \\
\mathrm{SC}, \mathrm{SP}\end{array}$ & $\begin{array}{l}\text { FERNANDES et al. (2020a); } \\
\text { SHIMBORI et al. (2014) }\end{array}$ \\
\hline \multicolumn{4}{|l|}{ Nesomesochorinae } \\
\hline Nonnus Cresson, 1874 & 1 & $\begin{array}{l}\text { AM, CE, ES, GO, MT, PI, } \\
\text { PR, RJ, RN, RS, SP }\end{array}$ & $\begin{array}{l}\text { AZEVEDO et al. (2015); FERNANDES et } \\
\text { al. (2020a); FERNANDES et al. (2020b); } \\
\text { KUMAGAl; GRAF (2000) }\end{array}$ \\
\hline \multicolumn{4}{|l|}{ Ophioninae } \\
\hline Enicospilus Stephens, 1835 & 5 & $\begin{array}{c}\text { AL, AM, BA, CE, DF, ES, } \\
\text { GO, MG, MS, MT, PB, PE, PI, } \\
\text { PR, RJ, RN, RO, RS, SC, SP }\end{array}$ & FERNANDES et al. (2014) \\
\hline
\end{tabular}


Table 3. Continuation.

\begin{tabular}{|c|c|c|c|}
\hline Taxon & Morphospecies & Distribution & References \\
\hline \multicolumn{4}{|l|}{ Orthocentrinae } \\
\hline Eusterinx Forster, 1869 & 2 & $\mathrm{AM}^{*}, \mathrm{PR}$ & KUMAGAI; GRAF (2000) \\
\hline $\begin{array}{l}\text { Orthocentrus } \\
\text { Gravenhorst, } 1829\end{array}$ & 3 & $\begin{array}{l}\mathrm{AM}^{*}, \mathrm{BA}, \mathrm{ES} \\
\mathrm{PB}, \mathrm{PR}, \mathrm{RN}, \mathrm{SC}\end{array}$ & $\begin{array}{l}\text { AZEVEDO et al. (2015); FERNANDES et al. } \\
\text { (2019); FERNANDES et al. (2O2Oa); KUMAGAI; } \\
\text { GRAF (2000); SHIMBORI et al. (2014) }\end{array}$ \\
\hline Plectiscidea Viereck, 1914 & 1 & $\mathrm{AM}^{* *}$ & \\
\hline \multicolumn{4}{|l|}{ Tersilochinae } \\
\hline Stethantyx Townes, 1971 & 1 & $\begin{array}{c}\mathrm{AM}^{*}, \mathrm{BA}, \mathrm{ES} \\
\mathrm{MG}, \mathrm{PR}, \mathrm{RJ}, \mathrm{SC}, \mathrm{SP}\end{array}$ & $\begin{array}{l}\text { AZEVEDO et al. (2015); FERNANDES et al. } \\
\text { (2019); FERNANDES et al. (2020a) }\end{array}$ \\
\hline \multicolumn{4}{|l|}{ Tryphoninae } \\
\hline Netelia Gray, 1860 & 1 & $\begin{array}{l}\text { AM, BA, CE, ES, GO, MS, } \\
\text { MT, PB, PE, PR, RJ, RN, SC }\end{array}$ & $\begin{array}{l}\text { FERNANDES et al. (2020a); FERNANDES et al. } \\
\text { (2020b); KUMAGAI; GRAF (2000); SHIMBORI } \\
\text { et al. (2014) }\end{array}$ \\
\hline
\end{tabular}

*New records for the state of Amazonas; **new record for Brazil; AL: Alagoas; AP: Amapá; AM: Amazonas; BA: Bahia; CE: Ceará; DF: Distrito Federal; ES: Espírito Santo; GO: Goiás; MA: Maranhão; MT: Mato Grosso; MS: Mato Grosso do Sul; MG: Minas Gerais; PA: Pará; PB: Paraíba; PR: Paraná; PE: Pernambuco; PI: Piaú́; RJ: Rio de Janeiro; RN: Rio Grande do Norte; RS: Rio Grande do Sul; RO: Rondônia; RR: Roraima; SC: Santa Catarina; SP: São Paulo; TO: Tocantins.

by FERNANDES et al. (2019), 36 genera were registered for the state. In a study conducted by AZEVEDO et al. (2015) in Espírito Santo State, with a sampling of over two years of collection, 100 genera were registered for the state. In a survey conducted in Mato Grosso do Sul State, only 20 genera were registered (SHIMBORI et al., 2017).

In this study, most of the genera registered for the first time in Amazonas State were sampled in organic crop managements of guarana. This indicates that the intensity of management affects the fauna of Ichneumonidae and that organic management apparently contributes to the maintenance of different genera in this system, which could have been better confirmed by conducting similar studies on a larger number of agricultural properties.

\section{CONCLUSION}

This study facilitated the characterization of the Ichneumonidae assemblage present in the conventional and organic guarana (Paullinia cupana) cultivation, in an experimental area of EMBRAPA — Manaus City (Amazonas State, Brazil), highlighting the high number of rare, less frequent and accidental genera, and revealing the predominant groups in each management system. Moreover, the present study elevated the number of known subfamilies for Amazonas, from 13 to 18, as well as the number of known genera, from 50 to 60 . The results obtained are hopefully expected to support and pave the way for further studies related to the biology and ecology of the group in agroecosystems.

ACKNOWLEDGEMENTS: The authors would like to thank Conselho Nacional de Desenvolvimento Científico e Tecnológico (CNPq) for granting scholarship; Programa de Capacitação Institucional” - PCl/CNPq (process n $312879 / 2019-9$ ) and “Coordenação de Aperfeiçoamento de Pessoal de Nível Superior” (CAPES), Brasil- Finance Code 001.

FUNDING: This study was funded by Fundação de Amparo à Pesquisa do Amazonas - FAPEAM, Universal Amazonas (process $n^{\circ}$ 062.00770/2015).

CONFLICTS OF INTEREST: The authors certify that they have no commercial or associative interest that represents a conflict of interest in connection with the manuscript.

AVAILABILITY OF DATA AND MATERIAL: The datasets generated and/or analyzed during the current study are available from the corresponding author.

AUTHORS' CONTRIBUTIONS: Conceptualization: ANTUNES, N.T.B.; FERNANDES, D.R.R. Data curation: ANTUNES, N.T.B.; FERNANDES, D.R.R. Formal analysis: ANTUNES, N.T.B.; FERNANDES, D.R.R. Funding acquisition: FERNANDES, D.R.R. Investigation: ANTUNES, N.T.B.; FERNANDES, D.R.R. Methodology: ANTUNES, N.T.B.; FERNANDES, D.R.R. Project administration: FERNANDES, D.R.R. Resources: FERNANDES, D.R.R. Supervision: FERNANDES, D.R.R. Validation: FERNANDES, D.R.R; ANTUNES, N.T.B. Visualization: ANTUNES, N.T.B. Writing - original draft: ANTUNES, N.T.B.; FERNANDES, D.R.R. Writing - review \& editing: FERNANDES, D.R.R.; ANTUNES, N.T.B. 


\section{REFERENCES}

AGUIAR, A.P.; SANTOS, B.F. Biological synopsis of Photocryptus Viereck (Hymenoptera, Ichneumonidae, Cryptinae), with eight new host records and fourteen new geographic records. Zootaxa, v.2148, n.1, p.49-54, 2009. http://dx.doi.org/10.11646/ zootaxa.2148.1.4

AZEVEDO, C.O.; DAL MOLIN, A.; PENTEADO-DIAS, A.; MACEDO, A.C.C.; RODRIGUEZ, B.; DIAS, B.Z.K.; WAICHERT, C.; AQUINO, D.; SMITH, D. R.; SHIMBORI, E.M.; NOLL, F.B.; GIBSON, G.; ONODY, H.C.; CARPENTER, J.M.; LATTKE, J.E.; RAMOS, K.S.; WILLIAMS, K.; MASNER, L.; KIMSEY, L.S.; TAVARES, M.T.; OLMI, M.; BUFFINGTON, M.L.; OHL, M.; SHARKEY, M.; JOHNSON, N.F.; KAWADA, R.; GONÇALVES, R.B.; FEITOSA, R.M.; HEYDON, S.; GUERRA, T.M.; SILVA, T.S.R.; COSTA, V. Checklist of the genera of Hymenoptera (Insecta) from Espirito Santo. Boletim do Museu Biológico Mello Leitão, v.37, n.3, p.313-343, 2015.

BENNETT, A.M.R.; CARDINAL, S.; GAULD, I.D.; WAHL, D.B. Phylogeny of the subfamilies of Ichneumonidae (Hymenoptera). Journal of Hymenoptera Research, v.71, p.1-156, 2019. http://dx.doi. org/10.3897/jhr.71.32375

CODDINGTON, J.A.; AGNARSSON, I.; MILLER, J.A.; KUNTNER, M.; HORMIGA, G. Undersampling bias: the null hypothesis for singleton species in tropical arthropod surveys. Journal of Animal Ecology, v.78, n.3, p.573-584, 2009. http://dx.doi. org/10.1111/j.1365-2656.2009.01525.x

COMÉRIO, E.F.; ONODY, H.C.; BENASSI, V.L.R.M. Levantamento da fauna de Ichneumonidae (Hymenoptera) em cultivo de coqueiro anão verde associado às plantas invasoras. EntomoBrasilis, v.5, n.2, p. 109-1 14, 2012. http://dx.doi.org/10.12741/ebrasilis. v5i2.196

DAJOZ, R. (Ed.). Ecologia Geral. Petrópolis: Vozes, 1973. 472p.

ESTRADA, C.I.N. (Ed.). Control biológico de insectos: um enfoque agroecológico. Medellín: Editorial Universidad de Antioquia, 2008. 282p.

FERNANDES, D.R.R.; ONODY, H.C.; LARA, R.I.R.; PERIOTO, N.W. Annotated Checklist of Brazilian Ophioninae (Hymenoptera: Ichneumonidae). EntomoBrasilis, v.7, n. 2, p. 124-133, 2014. http://dx.doi.org/10.12741/ebrasilis.v7i2.330

FERNANDES, D.R.R.; SANTOS, J.J.M.; LARA, R.I.R.; SILVA JUNIOR, J.C.; FERREIRA, H.A.; PERIOTO, N.W. Fauna de Ichneumonidae (Hymenoptera: Ichneumonoidea) em áreas de Caatinga do Sudoeste da Bahia, Brasil. EntomoBrasilis, v.12, n. 3, p. 126-131, 2019. http://dx.doi.org/10.12741/ ebrasilis.v $12 \mathrm{i} 3.837$

FERNANDES, D.R.R; SANTOS, B.F.; PÁDUA, D.G.; ARAUJO, R.O Ichneumonidae in Catálogo Taxonômico da Fauna do Brasil. PNUD. 2020. Disponível em: http://fauna.jbrj.gov.br/fauna/ faunadobrasil/2248. Access on: Mar. 102020.

FERNANDES, D.R.R.; ANTUNES, N.T.B.; ARAUJO, E.L.; LARA, R.I.R.; PERIOTO, N.W. Hymenoptera fauna, with emphasis on
Ichneumonidae from an area of Caatinga in Northeast Brazil. EntomoBrasilis, v. 13, e0874, 2020b. https://doi.org/10.12741/ ebrasilis.v13.e0874

FERNÁNDEZ-TRIANA, J.L.; RAVELO, H.G. A taxonomic review of Cuban Eiphosoma Cresson (Hymenoptera: Ichneumonidae) with biogeographical notes. Zootaxa, v. 1655, p.49-61, 2007. http://dx.doi.org/10.1 1646/zootaxa.1655.1.2

GAULD, I.D. An introduction to the Ichneumonidae of Australia; with a contribuition of Metopiina by M.G Fitton. London: British Museum (Natural History), 1984. 413p.

GAULD, I.D. A survey of the Ophioninae (Hymenoptera: Ichneumonidae) of tropical Mesoamerica with special reference to the fauna of Costa Rica. Bulletin of the British Museum (Natural History), Entomology series, London, v.57, p.1-309, 1988.

GAULD, I.D. The Ichneumonidae of Costa Rica, 1. Introduction, keys to subfamilies, and keys to the species of the lower Pimpliform subfamilies Rhyssinae, Poemeniinae, Acaenitinae and Cylloceriinae. Memoirs of the American Entomological Institute (Gainesville), v.47, p.1-589, 1991.

GAULD, I.D. The Ichneumonidae of Costa Rica, 3. Introduction and keys to species of the subfamilies Brachycyrtinae, Cremastinae, Labeninae and Oxytorinae, with an appendix on the Anomaloninae. Memoirs of the American Entomological Institute, v.63, p. 1-453, 2000.

GAULD, I.D. Familia Ichneumonidae. In: HANSON, P. E.; GAULD, I. D. (Eds.). Hymenoptera de la Region Neotropical. Memoirs of the American Entomological Institute, v.77, p.1-994, 2006.

GAULD, I.D.; MITCHELL, P.A. The taxonomy, distribution and host preferences of African parasitic wasps of the subfamily Ophioninae. London: Commonwealth Institute of Entomology, 1978. 287p.

GAULD, I.D.; MITCHELL, P.A. The taxonomy, distribution and host preferences of Indo-Papuan parasitic wasps of the subfamiliy Ophioninae. Londres: Commonwealth Institute of Entomology, $1981.611 \mathrm{p}$.

GONDIN, C.J.E. Alguns aspectos da biologia reprodutiva do guaranazeiro (Paullinia cupana var. sorbilis (Mart.) Ducke Sapindaceae. Acta Amazônica, v.14, n.1-2, p.9-38. 1984. http:// dx.doi.org/10.1590/1809-43921984142038

GONZÁLEZ, R.C.; BERRIO, A.R. Diversidad de avispas parasitoides (Hymenoptera) en agroecosistemas de palto (Persea americana Mill.) y mandarina (Citrus spp.) en Cañete, Lima, Perú. Aporte Santiaguino, v.8, n.2, p.207-218, 2015.

GONZÁLEZ-MORENO, A.; BORDERA, S. Review of Mexican Species of Podogaster Brull, (Hymenoptera: Ichneumonidae: Anomaloninae) with Description of Two New Species. Neotropical Entomology, v.42, p.39-51, 2013. http://dx.doi.org/10.1007/ s13744-012-0082-4 
GUERRA, T.M.; PENTEADO-DIAS, A.M. Abundância de Ichneumonidae (Hymenoptera) em área de mata em São Carlos, Estado de São Paulo, Brasil. Acta Scientiarum: Biological Sciences, v.24, n.2, p.363-368, 2002. http://dx.doi.org/10.4025/actascibiolsci. v24i0.2294

HAMMER, O.; HARPER, D.A.T.; RIAN, P.D. Past: Palaeonthological statistics software package for education and data analysis. Versão. 3,18, 2001 . Available from: https://folk.uio.no/ohammer/past/. Access on: Dec. 122017.

HANSON, P.E.; GAULD, I.D. Hymenoptera de la Región Neotropical. Memoirs of the American Entomological Institute, Gainesville, v.77, p.1-994, 2006

HOLDEFER, D.R.; GARCIA, F.R.M. Análise faunística de cerambicídeos (Coleoptera, Cerambycidae) em floresta subtropical úmida brasileira. Entomotropica, v.30, n.13, p.118-134, 2015.

INSTITUTO BRASILEIRO DE GEOGRAFIA E ESTATÍSTICA (IBGE). Levantamento sistemático da produção agrícola. v.30. n.12. Rio de Janeiro: IBGE, 2017. p.1-82. Disponível em: ftp://ftp.ibge. gov.br/Producao_Agricola/Levantamento_Sistematico_da_ Producao_Agricola_[mensal]/Fasciculo_Indicadores_IBGE/2017/ estProdAgr_201712.pdf. Access on: Jun. 272018.

KASPARYAN, D.R.; RUÍZ-CANCINO, E. Cryptini de México (Hymenoptera: Ichneumonidae: Cryptinae) Parte II, Serie Avispas Parasíticas de Plagas y otros Insectos n.2. Cd. Victoria, Tamaulipas, México: Universidad Autónoma de Tamaulipas, 2008. 373p.

KHALAIM, A.I.; RUIZZ-CANCINO, E. Mexican species of the genus Stethantyx Townes (Hymenoptera, Ichneumonidae, Tersilochinae). ZooKeys, v.360, p.83-94, 2013. http://dx.doi.org/10.3897/ zookeys.360.6362

KUMAGAI, A.F. Os Ichneumonidae (Hymenoptera) da Estação Ecológica da Universidade Federal de Minas Gerais, Belo Horizonte, com ênfase nas espécies de Pimplinae. Revista Brasileira de Entomologia, v.46, n.2, p.189-194, 2002. http://dx.doi. org/10.1590/S0085-56262002000200011

KUMAGAI, A.F.; GRAF, V. Ichneumonidae (Hymenoptera) de áreas urbana e rural de Curitiba, Paraná, Brasil. Acta Biológica Paranaense, v.29, n.1-4, p.153-168, 2000. http://dx.doi. org/10.5380/abpr.v29i0.588

KUMAGAI, A.F.; GRAF, V. Biodiversidade de Ichneumonidae (Hymenoptera) e monitoramento das espécies de Pimplinae e Poemeniinae do Capão da Imbuia, Curitiba, Paraná. Revista Brasileira de Zoologia, v.19, n.2, p.445-452. 2002. http://dx.doi. org/10.1590/SO101-81752002000200010

LUDWIG, J.A.; REYNOLDS J.F. Statistical Ecology. A Primer on methods and computing. New York: John Wiley e Sons, INC., 1988. 337p.

MOERICKE, V. Eine Farbfalle zur Kontrolle des Fluges von Blattläusen, insbesondere der Pfisichblattlaus Myzodes persicae (Sulz.). NachrB 1. dtsch. PfISchDienst, Stuttgart, v.3, p.23, 1951.

MORAES, R.C.B.; HADDAD, M.L.; SILVEIRA-NETO, S; REYES, A.E.L. Software para análise faunística - AnaFau. In: SIMPÓSIO DE
CONTROLE BIOLÓGICO, 8., 2003. Resumos... São Pedro, 2003. 195p. Available from: http://www.lea.esalq.usp.br/softwares. Access on: Feb. 022017.

NOVOTNÝ, V.; BASSET, Y. Rare species in communities of tropical insect herbivores: pondering the mystery of singletons. Oikos, v.89, n.3, p.564-572, 2000. http://dx.doi. org/10.1034/j.1600-0706.2000.890316.x

NOYES, J.S. A study of five methods of sampling Hymenoptera (Insecta) in a tropical rainforest, with special reference to the Parasitica. Journal of Natural History, v.23, n.2, p.285-298, 1989. http://dx.doi.org/10.1080/00222938900770181

PÁDUA, D.G.; ZAMPIERON, S.L.M.; NUNES, J.F. Composition of the Families of Parasitoids Wasp in the Parque Nacional da Serra da Canastra, Minas Gerais, Brazil. EntomoBrasilis, v.7, n.3, p. 199-206, 2014. http://dx.doi.org/10.12741/ebrasilis.v7i3.443

PALACIO, E.E.; WAHL, D.B. Familia Ichneumonidae. In: FERNÁNDEZ, F.; SHARKEY, M. J. (Eds.). Introducción a los Hymenoptera de la Región Neotropical. Bogotá: Sociedad Colombiana de Entomología y Universidad Nacional de Colombia, 2006. p.293-330.

PEREIRA, J.C.R. Cultura do guaranazeiro no Amazonas. $4^{\text {a ed. }}$ Manaus: Embrapa Amazônia Ocidental, 2005. $21 \mathrm{p}$.

PERIOTO, N.W.; LARA, R.I.R.; SANTOS, J.C.C.; SELEGATTO, A. Himenópteros parasitóides (Insecta, Hymenoptera) coletados em cultura de algodão (Gossypium hirsutum L.) (Malvaceae), no município de Ribeirão Preto, SP, Brasil. Revista Brasileira de Entomologia, v.46, n.2, p.165-168, 2002. http://dx.doi. org/10.1590/S0085-56262002000200008

PFIFFNER, L.; LUKA, A.H. Overwintering of arthropods in soils of arable fields and adjacent semi-natural habitats. Agriculture, Ecosystem \& Environment, v.78, n.3, p.215-222, 2000. https:// doi.org/10.1016/S0167-8809(99)00130-9

SANDONATO, D.L.; ONODY, H.C.; PENTEADO-DIAS, A.M. Fauna de Campopleginae (Hymenoptera, Ichneumonidae) em hortas orgânicas em Araraquara e São Carlos, SP, Brasil. Biota Neotropica, v.10, n.2, p.117-121, 2010. https://doi.org/10.1590/ S1676-06032010000200014

SCHERRER, M.V.; AGUIAR, A.P. A review of Debilos Townes (Hymenoptera, Ichneumonidae, Cryptinae) with description of twenty-seven new species. Zootaxa, v.3469, p.1-76, 2012. https://doi.org/10.1 1646/zootaxa.3469.1.1

SCHOENINGER, K.; SOUZA, J.L.P.; KRUG, C.; OLIVEIRA, M.L. Diversity of parasitoid wasps in conventional and organic guarana (Paullinia cupana var. sorbilis) cultivation areas in the Brazilian Amazon. Acta Amazonica, v.49, p. 283-293, 2019. http:// dx.doi.org/10.1590/1809-4392201804560

SHIMBORI, E.M.; LOFFREDO, A.P.S.; CASTRO, C.S.; BORTONI, M.A.; PENTEADO-DIAS, A.M. Contribuição ao conhecimento da fauna de Ichneumonoidea (Hymenoptera) do Semiárido brasileiro. In: BRAVO, F.; CALOR, A. (Eds.) Artrópodes do Semiárido: biodiversidade e conservação. Feira de Santana: Print Mídia, 2014. p.139-152.

SHIMBORI, E.M; ONODY, H.C; FERNANDES, D.R.R.; SILVESTRE, R.; TAVARES M.T.; PENTEADO-DIAS, A.M. Hymenoptera 
"Parasitica" in the state of Mato Grosso do Sul, Brazil. Iheringia, Série Zoologia, 107(Supl.), p.e2017121, 2017. https://doi. org/10.1590/1678-4766e2017121

SILVEIRA-NETO, S.; NAKANO, O.; BARDIN, D.; VILANOVA, N.A. Manual de ecologia dos insetos. São Paulo: Agronômica Ceres, 1976. 420p.

TANQUE, R.L.; KUMAGAI, A.F.; FRIEIRO-COSTA, F.A.; SOUZA, B. Ichneumonidae (Insecta: Hymenoptera) da Reserva do Boqueirão, Ingaí - MG. Revista Brasileira de Zoociências, v. 12 , n.3, p.241-247, 2010

TANQUE, R.L;; KUMAGAI, A.F; SOUZA, B. KORASAKI, V. Structure and dynamics of the taxocenoses of Pimplinae, Poemeniinae, Rhyssinae, Anomaloninae and Metopiinae in an urban secondary semideciduous montane forest. Neotropical Entomology, v.44, n.3, p.224-231, 2015. https://doi.org/10.1007/s13744-015-0275-80

TEDESCO, A.M.; AGUIAR, A.P. Phylogeny and Revision of Toechorychus Townes (Hymenoptera, Ichneumonidae,
Cryptinae), with descriptions of thirty-five new species. Zootaxa, v.3633, n.1, p.1-138, 2013. https://doi.org/10.11646/ zootaxa.3633.1.1

TEMPEST, A.M.; BERGMANN, E.C.; FARIA, A.M; PENTEADO-DIAS, A.M; IMENES, S.D.L.; GUERRA, T.M. Sobre os Ichneumonidae presentes em cultura de seringueira (Hevea brasiliensis). Arquivos do Instituto Biológico, v.65, n.2, p.63-68, 1998.

TOWNES, H. A light-weight Malaise trap. Entomological News, v.83, p.239-247, 1972.

WILLIAMS, R.N.; WEISS, M.J.; KEHAT, M.; BLUMBERG, D. The hymenopterous parasites of Nitidulidae. Phytoparasitica, v.12, p.53-64, 1984. https://doi.org/10.1007/BF02980798

YU, D.S.; ACHTERBERG, C.; HORSTMANN, K. Taxapad 2016, Ichneumonoidea 2015. Database on flash-drive. Nepean, Ontario, Canada. Available from: www.taxapad.com. Access on: Jan. 212018. 